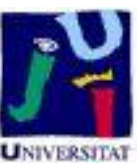

Título artículo / Títol article: Synthesis and Leishmanicidal Activity of Cinnamic Acid Esters: Structure-Activity Relationship

Autores / Autors

Otero, Elver ; Robledo, Sara M. ; Díaz Oltra, Santiago ; Carda Usó, Miguel ; Muñoz, Diana ; Paños Pérez, Julián ; Vélez, Ivan D. ; Cardona, Wilson

Revista:

Medicinal Chemistry Research

Versión / Versió:

Pre-print

Cita bibliográfica / Cita bibliogràfica (ISO 690):
OTERO, Elver, et al. Synthesis and leishmanicidal activity of cinnamic acid esters: Structure-activity relationship. Medicinal Chemistry Research, 2014, vol. 23, no 3, p. 1378-1386. 


\title{
Synthesis and Leishmanicidal Activity of Cinnamic Acid Esters: Structure-Activity Relationship
}

Elver Otero $^{\mathrm{a}}$, Sara Robledo ${ }^{\mathrm{b}}$, Santiago Díaz $^{\mathrm{c}}$, Miguel Carda ${ }^{\mathrm{c}}$, Diana Muñoz ${ }^{\mathrm{b}}$, Julian Paños ${ }^{\mathrm{c}}$, Ivan D. Vélez ${ }^{\mathrm{b}}$ and Wilson Cardona ${ }^{\mathrm{a}^{*}}$

a. Instituto de Química, Química de Plantas Colombianas, Universidad de Antioquia, A.A 1226, Medellín, Colombia.

b. Programa de Estudio y Control de Enfermedades Tropicales (PECET), Universidad de Antioquia, A.A 1226, Medellín, Colombia.

c. Departamento de Quimica Inorganica y Organica, Universidad Jaume I, E-12071 Castellon, Spain.

\begin{abstract}
Several cinnamic acid esters were obtained via Fischer esterification of cinnamic acids derivatives with aliphatic alcohols. Cinnamic acids derivatives were synthesized via Knoevenagel reaction between substituted benzaldehydes and malonic acid in aqueous medium assisted by microwave heating. Structures of the products were elucidated by spectroscopic analysis. The synthesized compounds were evaluated for antileishmanial activity against L. panamensis amastigotes and cytotoxic activity against U-937 cells. The compounds 6, 10-12 and 18, were active against Leishmania parasite but toxic for mammalian cells. They are potential candidates for antileishmanial drug development.
\end{abstract}

Keywords Leishmaniasis, Antiprotozoal, Caffeic acid, Cinnamic acid esters

* Corresponding author. Tel: 05742195653. Fax: 05742330120. E-mail: wcardona@matematicas.udea.edu.co 


\section{Introduction}

Leishmaniasis is one of the world's most neglected diseases, affecting largely the poorest of the poor, mainly in developing countries; 350 million people are considered at risk of contracting leishmaniasis, and some 2 million new cases occur yearly (Murray et al., 2005) and WHO has classified leishmaniasis as a category 1 disease, i.e. emerging and uncontrolled (http://www.who.int/topics/leishmaniasis/en/). This disease has symptoms from skin lesions to fatal systemic infection caused by protozoan parasites of the Leishmania species (Handman 1999). Recently, a dramatic increase in the number of cases of leishmaniasis has been observed in patients with compromised T-cell function, such as those infected with the human immunodeficiency virus (Wolday et al., 1999). Drugs currently in use as the antimony derivative glucantine, the bis-amidines, pentamidine and stilbamidine or the glycomacrolide amphotericin B, display high liver and heart toxicities, develop clinical resistance after a few weeks of treatment, and moderate and severe side effects (Desjeux et al., 2004; Ouellette et al., 2004; Barrett et al,. 2002; Croft et al., 2003; Faraut-Gambarelli et al., 1997; Olliaro et al,. 1993). For these reasons it becomes necessary to discover new, more potent and selective agents for treating this increasing parasitosis.

Caffeic acid (1), 3,4-dihydroxy cinnamic acid, and its esters derivatives exhibit a broad spectrum of biological activities, including anti-inflammatory (Jayaprakasam et al., 2006; Da Cunha et al., 2004), antimicrobial (Almajano et al., 2007; Noriaki et al., 2005; King et al., 1999; Valenta et al., 1998; Bowles et al., 1994) antioxidant (Hung et al., 2005; Noriaki et al., 2005; Kikuzaki et al,. 2002; Son et al., 2002; Rajan et al., 2001) and anticarcinogenic effects (De et. al. 2011). Besides, some studies showed high leishmanicidal activity for these compounds ( $\mathrm{IC}_{50} 4.4 \mathrm{nM}$ to caffeic acid (Radtke et al., 2003) and 2.0, 10 and $1.8 \mu \mathrm{M}$ to 2, 3 and 4 esters (fig. 1) (Cabanillas et al., 2010)). In the search of new therapeutic alternatives for the treatment of Leishmaniasis several cinnamic acid esters analogues were synthesized and their cytotoxic and leishmanicidal activities were determined.
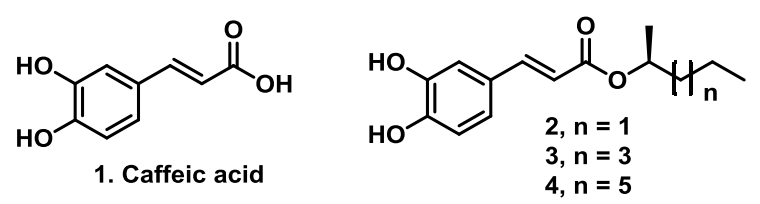

Fig. 1 Caffeic acid and its esters derivatives 


\section{Results and Discussion}

Chemistry

Cinnamic acid esters were obtained via Knoevenagel condensation in water under microwave heating (Gupta et al., 2007). These compounds were dissolved in thionyl chloride; the solution was stirred and refluxed by two hours. After evaporation, the acid chloride was added to a solution of cetyl alcohol in dichloromethane. Following evaporation and purification by column chromatography a total of six compounds were obtained. The cetyl alcohol was change by dodecyl alcohol or stearyl alcohol (Narasimhan et al., 2004). 3,4-dimethoxycinnamic acid was dissolved in methanol, propanol, pentanol, hexanol and octanol; acetyl chloride was added and the solution was stirred under reflux to complete the reaction. Following evaporation and purification by column chromatography a total of five compounds were obtained (De Campos et al., 2009). Compounds 17 and 18 were similarly obtained by starting from cinnamic and caffeic acid, respectively. Compound 16 was obtained by catalytic hydrogenation of compound 15 (Cardona et al., 2006) (scheme 1).

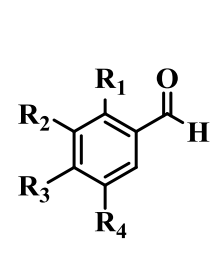

$\mathbf{R}_{1}=\mathbf{R}_{\mathbf{2}}=\mathbf{H}, \mathbf{R}_{\mathbf{3}}=\mathbf{R}_{\mathbf{4}}=\mathrm{OCH}_{3}$

$\mathbf{R}_{1}=\mathbf{R}_{\mathbf{2}}=\mathrm{OCH}_{3}, \mathbf{R}_{\mathbf{3}}=\mathbf{R}_{\mathbf{4}}=\mathbf{H}$

$\mathbf{R}_{1}=\mathbf{R}_{3}=\mathrm{OCH}_{3}, \mathbf{R}_{\mathbf{2}}=\mathbf{R}_{\mathbf{4}}=\mathbf{H}$

$\mathbf{R}_{1}=\mathbf{R}_{4}=\mathrm{OCH}_{3}, \mathbf{R}_{2}=\mathbf{R}_{3}=\mathbf{H}$

$\mathbf{R}_{\mathbf{1}}=\mathbf{R}_{\mathbf{2}}=\mathbf{R}_{\mathbf{4}}=\mathbf{H}, \mathbf{R}_{\mathbf{3}}=\mathrm{OCH}_{3}$

10. $n=9, R_{1}=R_{2}=H, R_{3}=R_{4}=O_{3}$

11. $n=15, R_{1}=R_{2}=H, R_{3}=R_{4}=O_{3}$

$\mathbf{n}=\mathbf{1 3}$

12. $R_{1}=R_{2}=H, R_{3}=R_{4}=O C H_{3}$

13. $R_{1}=R_{2}=O O_{3}, R_{3}=R_{4}=H$

14. $R_{1}=R_{4}=O O_{3}, R_{2}=R_{3}=H$

15. $\mathbf{R}_{1}=\mathbf{R}_{\mathbf{2}}=\mathbf{R}_{\mathbf{4}}=\mathrm{H}, \mathbf{R}_{\mathbf{3}}=\mathrm{OCH}_{3}$
Malonic acid $\mathrm{TBAB}, \mathrm{K}_{2} \mathrm{CO}_{3}, \mathrm{H}_{2} \mathrm{O}$ MW<smiles>[R4]c1cc(/C=C/C(=O)O)c([R1])c([R4])c1[R4]</smiles>
to $\mathbf{R}_{\mathbf{1}}=\mathbf{R}_{\mathbf{2}}=\mathbf{H}$,

$\mathbf{R}_{\mathbf{3}}=\mathbf{R}_{\mathbf{4}}=\mathrm{OCH}_{\mathbf{3}}$

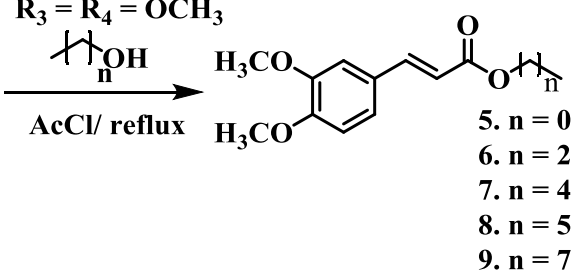

1. $\mathrm{SOCl}_{2}$

2. $\mathrm{H}_{\mathrm{n}} \mathrm{OH}$ $\mathbf{n}=9,13,15$
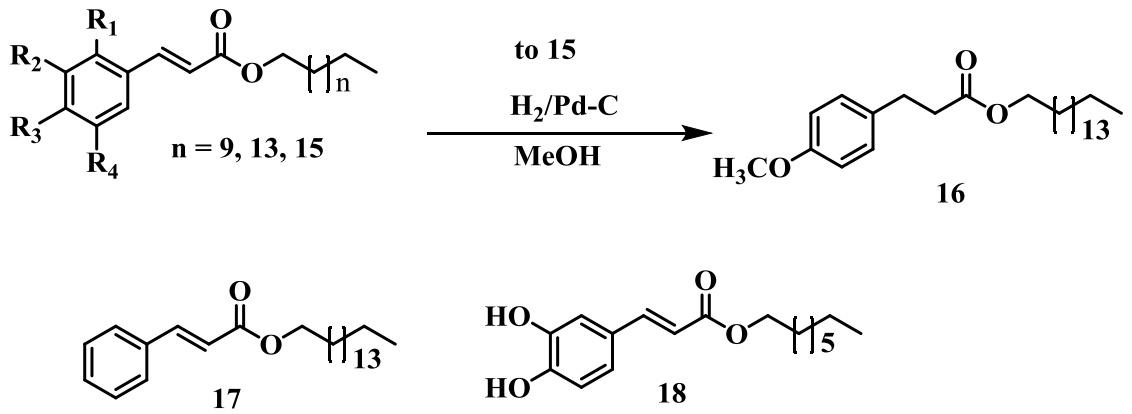

Scheme 1. Synthetic pathway to cinnamic acid esters derivatives 
The leishmanicidal activity and cytotoxicity of the synthesized compounds as well as amphotericin B, used as control drug, were evaluated following the method previously reported in the literature (Varela et al., 2009; Robledo et al., 2005; Weninger et al., 2001; Robledo et al., 1999). The results were reported as $\mathrm{EC}_{50}$ and $\mathrm{LC}_{50}$ values of compounds and are shown in the tables 1 and 2.

Table 1. In vitro leishmanicidal activity against axenic amastigotes of $L$. panamensis and toxicity of Cinnamic acid esters.

\begin{tabular}{|c|c|c|c|}
\hline Compound & $\begin{array}{c}\text { Cytotoxicity } \\
\text { U937 cells } \\
\mathrm{LC}_{50}(\mu \mathrm{g} / \mathrm{ml})^{\mathrm{a}}\end{array}$ & $\begin{array}{c}\text { Leishmanicidal } \\
\text { Activity } \\
\text { EC }_{50}(\mu \mathrm{g} / \mathrm{ml})^{\mathrm{b}}\end{array}$ & $\mathbf{S I}^{\mathbf{c}}$ \\
\hline 5 & $162.6 \pm 49.6$ & $55.0 \pm 14.7$ & 3.0 \\
\hline 6 & $85.3 \pm 17.5$ & $33.0 \pm 0.2$ & 2.6 \\
\hline 7 & $49.8 \pm 6.3$ & $38.1 \pm 9.7$ & 1.3 \\
\hline 8 & $54.7 \pm 7.7$ & $60.0 \pm 4.0$ & 0.9 \\
\hline 9 & $79.9 \pm 5.5$ & $>10 \overline{0} .0$ & $<0.8$ \\
\hline 10 & $49.7 \pm 18.1$ & $59.0 \pm 1.9$ & 0.8 \\
\hline 11 & $28.5 \pm 1.9$ & $108.2 \pm 1.5$ & 0.3 \\
\hline 12 & $69.1 \pm 6.7$ & $55.4 \pm 2.0$ & 1.2 \\
\hline 13 & $>200.0$ & $>100.0$ & $<2.0$ \\
\hline 14 & $>200.0$ & $>100.0$ & $<2.0$ \\
\hline 15 & $>200.0$ & $>100.0$ & $<2.0$ \\
\hline 16 & $>200.0$ & $>100.0$ & $<2.0$ \\
\hline 17 & $>200.0$ & $>100.0$ & $<2.0$ \\
\hline 18 & $9.9 \pm 1.7$ & $2.3 \pm 0.5$ & 4.3 \\
\hline Amphotericin B & $29.6 \pm 4.7$ & $0.05 \pm 0.01$ & 592 \\
\hline
\end{tabular}

According to the results shown in the table 1, only compounds 5, 6, 7, 8, 10, 12 and 18 showed activity against axenic amastigotes of $L$. panamensis with $\mathrm{EC}_{50}$ lower than $100 \mu \mathrm{g} / \mathrm{ml}$. The most active compound was $18\left(\mathrm{EC}_{50}=2.3 \mu \mathrm{g} / \mathrm{ml}\right)$ followed by $\mathbf{6}$ and 7 exhibiting $\mathrm{EC}_{50}$ values of 33.0 and 38.1 $\mu \mathrm{g} / \mathrm{ml}$, respectively. Compounds 5, 8, 10 and 12 showed a moderate leishmanicidal activity with an $\mathrm{EC}_{50}$ ranging between 55.0 and $60.0 \mu \mathrm{g} / \mathrm{ml}$. On the other hand, a high toxicity activity was measured for compounds 6 to 12 and 18, with a $\mathrm{LC}_{50}<100 \mu \mathrm{g} / \mathrm{ml}$. The lower toxic activity was obtained with the compound $5\left(\mathrm{EC}_{50}=162.6 \mu \mathrm{g} / \mathrm{ml}\right)$. No leishmanicidal activity and toxicity were detected for the compounds 13 to 17 ( $\mathrm{EC}_{50}$ values higher than $100 \mu \mathrm{g} / \mathrm{ml}$ and $\mathrm{LC}_{50}$ higher than $200 \mu \mathrm{g} / \mathrm{ml}$, respectively). The best selectivity index was observed for compounds 18, 5 and $\mathbf{6}$ with values of 4.3, 3.0 and 2.6, respectively. Compound $\mathbf{1 8}$ is structurally similar to the compound 4 reported by Cabanillas et al. (2010). Although the $\mathrm{EC}_{50}$ and $\mathrm{LC}_{50}$ values reported previously for compound 4 were slightly different, $\left(\mathrm{EC}_{50}=0.55 \mu \mathrm{g} / \mathrm{mL}\right.$ and $\left.\mathrm{LC}_{50} 3.9 \mu \mathrm{g} / \mathrm{mL}\right)$, both compounds are cytotoxic and highly active against Leishmania parasites. Differences in the Leishmania species and cell type used in the 
assays could explain the different results obtained with compounds $\mathbf{1 8}$ and $\mathbf{4}$. Thus, compound $\mathbf{4}$ was tested against axenic amastigotes of L. amazonensis and murine peritoneal macrophages (Cabanillas et al., 2010) whereas compound 18 was tested in this report against axenic amastigotes of L. panamensis and human macrophages.

Structure-activity relationship

There is a relationship between the leishmanicidal activity and compounds structure. The smaller the alkyl chains the higher the selectivity index (5, 6 vs 7-12); the degree of oxygenation is essential for activity, primarily in positions 2,3 and 4 (12 vs 13-17); hydroxyl groups increase both the activity and cytotoxicity ( 9 vs 18); The importance of the double bond is not conclusive since the compounds were inactive, which can be related to the degree of oxygenation (16 vs 17). However, if the action mechanism involves a Michael addition for nucleophilic amino acid residues, this would be crucial for the activity as has been reported for other $\alpha, \beta$-unsaturated compounds such as lactones, chalcones and cumarins (De Fatima et al., 2006; Buck et al., 2003).

Table 2. In vitro activity of Cinnamic acid esters against intracellular amastigotes of L. panamensis.

\begin{tabular}{ccc}
\hline \multirow{2}{*}{ Compound } & Leishmanicidal Activity & SI \\
& $\mathbf{E C}_{\mathbf{5 0}}(\boldsymbol{\mu} \mathbf{g} / \mathbf{m l})$ & \\
\hline $\mathbf{6}$ & $60.2 \pm 1.2$ & 2.6 \\
$\mathbf{1 0}$ & $25.2 \pm 2.3$ & 2.0 \\
$\mathbf{1 1}$ & $18.3 \pm 3.3$ & 1.6 \\
$\mathbf{1 2}$ & $26.5 \pm 2.0$ & 2.7 \\
$\mathbf{1 8}$ & $3.2 \pm 0.8$ & 3.1 \\
Amphotericin B & $0.06 \pm 0.01$ & 592 \\
\hline
\end{tabular}

The leishmanicidal activity against the intracellular forms of Leishmania parasites was also measured for all compounds. Only the compounds 6, 10-12 and 18 were active (table 2). The most active compounds were 18 and 11 with $\mathrm{EC}_{50}$ of 3.2 and $18.3 \mu \mathrm{g} / \mathrm{ml}$, respectively. The compound $\mathbf{6}$ had the lowest activity with $\mathrm{EC}_{50} 60.2 \mu \mathrm{g} / \mathrm{ml}$. The best SI was observed for compounds 18, 12 and 6 with values of 3.1, 2.7 and 2.6, respectively.

Overall, the compounds 6, 10,12 and 18 were apparently the most active compounds showing activity against both axenic and intracellular amastigotes of L. panamensis, while the compounds $\mathbf{5}, \mathbf{7}$ and $\mathbf{8}$ showed activity only on the axenic form of this Leishamania species.

\section{Conclusion}

The design, synthesis, and antileishmanial screening of fourteen cinnamic acid esters was reported. Several of the reported compounds have potential as leishmanicidal drugs, as determined by both the leishmanicidal activity and the cytotoxicity. The compounds 6, 10-12 and 18, active against Leishmania parasite but toxic for mammalian cells, are potential candidates for antileishmanial drug development. However, more studies on toxicity using other cell lines are needed in order to discriminate whether the toxicity shown by these compounds is against tumor or non-tumor cells.

\section{Experimental procedures}




\section{Chemistry}

IR spectra were recorded on a Perkin-Elmer Spectrum RX I FT-IR system in a KBr disk. ${ }^{1} \mathrm{H}$ NMR and ${ }^{13} \mathrm{C}$ NMR spectra were recorded on Varian Unity $500 \mathrm{MHz}$ spectrometer using $\mathrm{CDCl}_{3}$ as solvent and TMS as an internal standard. The chemical shifts are expressed in $\delta \mathrm{ppm}$. High resolution mass spectra were run by the electron impact mode (EIMS, $70 \mathrm{eV}$ ) on VG AutoSpec spectrometer. Silica gel 60 (Merck 0.063-0.200 mesh) was used for column chromatography, and precoated silica gel plates (Merck 60 F254 $0.2 \mathrm{~mm}$ ) were used for TLC.

\section{Synthesis of cinnamic acid esters (5-9)}

Cinnamic acid $(10 \mathrm{mmol})$, acetyl chloride $(0.5 \mathrm{mmol})$, and the alcohol $(50 \mathrm{ml})$, were placed in a $250 \mathrm{ml}$ 3-neck round- bottom flask equipped with a magnetic stirring bar. The mixture was stirred, heated to reflux for a period of 4 hours. The reaction mixture was concentrated on a rotatory evaporator, and the residue was purified by chromatographic column over silica gel eluted with a mixture hexane-ethyl acetate at different ratios to obtain the cinnamic acid esters in yield between $75-85 \%$.

\section{Synthesis of cinnamic acid esters (10-16)}

Cinnamic acid $(10 \mathrm{mmol})$ and thionyl chloride $(10 \mathrm{ml})$ were placed in a $50 \mathrm{ml} 3$-neck round-bottom flask equipped with a magnetic stirring bar. The mixture was stirred, heated to reflux for a period of 4 hours. The reaction mixture was concentrated on a rotatory evaporator, and the residue was added to a solution of cetyl alcohol (dodecyl or estearyl alcohol) in dichloromethane and the mixture was stirred and monitored by thin layer chromatography. The reaction was complete after about 4 hours. The mixture was transferred to a separatory funnel and then quenched by addition of a solution of $20 \mathrm{ml}$ of potassium carbonate; the organic layer was washed with water, separated, dried on anhydrous sodium sulfate, filtered and concentrated under reduced pressure. The residue was chromatographed over silica gel (hexane-ethyl acetate, different ratios) to obtain the cinnamic acid esters in yield between 70-85\%.

Methyl (2E)-3-(3,4-dimethoxyphenyl)prop-2-enoate (5)

Yield 74\%; yellow pale oil; IR ( $\left.\mathrm{KBr}, \mathrm{cm}^{-1}\right): v_{\max } 2945(\mathrm{C}-\mathrm{H}), 1700(\mathrm{C}=\mathrm{O}), 1627(\mathrm{C}=\mathrm{C}), 1510$ $\left(\mathrm{C}=\mathrm{C}_{\mathrm{Ar}}\right), 1270(\mathrm{C}-\mathrm{O}-\mathrm{C}), 1178((\mathrm{C}=\mathrm{O})-\mathrm{O}), 857\left(\mathrm{C}-\mathrm{H}_{\mathrm{Ar}}\right) ;{ }^{1} \mathrm{H} \mathrm{NMR}\left(\mathrm{CDCl}_{3}, 500 \mathrm{MHz}\right): \delta 3.78(3 \mathrm{H}, \mathrm{s}$, $\left.\mathrm{OCH}_{3}\right), 3.90\left(6 \mathrm{H}, \mathrm{s}, \mathrm{OCH}_{3}\right), 6.30(1 \mathrm{H}, \mathrm{d}, \mathrm{J}=15.9 \mathrm{~Hz},-\mathrm{CO}-\mathrm{CH}=), 6.86(1 \mathrm{H}, \mathrm{d}, \mathrm{J}=8.0 \mathrm{~Hz}, \mathrm{Ar}-\mathrm{H}), 7.04$ $(1 \mathrm{H}, \mathrm{d}, J=1.6 \mathrm{~Hz}, \mathrm{Ar}-\mathrm{H}), 7.10(1 \mathrm{H}, \mathrm{dd}, J=8.0,1.6 \mathrm{~Hz}, \mathrm{Ar}-\mathrm{H}), 7.63(1 \mathrm{H}, \mathrm{d}, J=15.9 \mathrm{~Hz}, \mathrm{Ar}-\mathrm{CH}=\mathrm{C})$; ${ }^{13} \mathrm{C} \mathrm{NMR}\left(\mathrm{CDCl}_{3}, 125 \mathrm{MHz}\right): \delta 51.46\left(\mathrm{OCH}_{3}\right), 55.83\left(\mathrm{OCH}_{3}\right), 55.91\left(\mathrm{OCH}_{3}\right), 109.50,110.88,115.51$ $(=\mathrm{C}-\mathrm{CO}-), 122.57,127.38,144.70(\mathrm{Ar}-\mathrm{C}=), 149.18,151.09,167.40(\mathrm{C}=\mathrm{O}) . \mathrm{MS}: \mathrm{m} / \mathrm{z} 223.0972(\mathrm{M}+$ 1).

Propyl (2E)-3-(3,4-dimethoxyphenyl)prop-2-enoate (6)

Yield 70\%; yellow pale oil; IR (KBr, cm $\left.{ }^{-1}\right): v \max 2967(\mathrm{C}-\mathrm{H}), 1706(\mathrm{C}=\mathrm{O}), 1635(\mathrm{C}=\mathrm{C}), 1513$ $\left(\mathrm{C}=\mathrm{C}_{\mathrm{Ar}}\right), 1260(\mathrm{C}-\mathrm{O}-\mathrm{C}), 1177((\mathrm{C}=\mathrm{O})-\mathrm{O}), 808\left(\mathrm{C}-\mathrm{H}_{\mathrm{Ar}}\right) ;{ }^{1} \mathrm{H} \mathrm{NMR}\left(\mathrm{CDCl}_{3}, 500 \mathrm{MHz}\right): \delta 0.97(3 \mathrm{H}, \mathrm{t}, J=$ $7.4 \mathrm{~Hz}), 1.70(2 \mathrm{H}, \mathrm{m}), 3.88\left(6 \mathrm{H}, \mathrm{s}, \mathrm{OCH}_{3}\right), 4.13(2 \mathrm{H}, \mathrm{t}, J=6.9 \mathrm{~Hz}), 6.29(1 \mathrm{H}, \mathrm{d}, J=16.0 \mathrm{~Hz},-\mathrm{CO}-$ $\mathrm{CH}=), 6.83(1 \mathrm{H}, \mathrm{d}, J=8.3 \mathrm{~Hz}, \mathrm{Ar}-\mathrm{H}), 7.03(1 \mathrm{H}, \mathrm{d}, J=1.8 \mathrm{~Hz}, \mathrm{Ar}-\mathrm{H}), 7.07(1 \mathrm{H}, \mathrm{dd}, J=8.3,1.6 \mathrm{~Hz}$, $\mathrm{Ar}-\mathrm{H}), 7.60(1 \mathrm{H}, \mathrm{d}, J=16.0 \mathrm{~Hz}, \mathrm{Ar}-\mathrm{CH}=\mathrm{C}) ;{ }^{13} \mathrm{C} \mathrm{NMR}\left(\mathrm{CDCl}_{3}, 125 \mathrm{MHz}\right): \delta 10.37\left(\mathrm{CH}_{3}\right), 22.02$ $\left(\mathrm{CH}_{2}\right), 55.79\left(\mathrm{OCH}_{3}\right), 55.86\left(\mathrm{OCH}_{3}\right), 65.94\left(-\mathrm{OCH}_{2}\right), 109.59,110.98,115.86$ (=C-CO-), 122.44, 127.35, $144.37(\mathrm{Ar}-\mathrm{C}=), 149.10,150.95,167.15(\mathrm{C}=\mathrm{O}) . \mathrm{MS}: \mathrm{m} / \mathrm{z} 251.1283(\mathrm{M}+1)$. 
Pentyl (2E)-3-(3,4-dimethoxyphenyl)prop-2-enoate (7)

Yield 85\%; yellow pale oil; IR (KBr, cm $\left.{ }^{-1}\right): v \max 2958(\mathrm{C}-\mathrm{H}), 1706(\mathrm{C}=\mathrm{O}), 1635(\mathrm{C}=\mathrm{C}), 1513$ $\left(\mathrm{C}=\mathrm{C}_{\mathrm{Ar}}\right), 1259(\mathrm{C}-\mathrm{O}-\mathrm{C}), 1160((\mathrm{C}=\mathrm{O})-\mathrm{O}), 807\left(\mathrm{C}-\mathrm{H}_{\mathrm{Ar}}\right) ;{ }^{1} \mathrm{H} \mathrm{NMR}\left(\mathrm{CDCl}_{3}, 500 \mathrm{MHz}\right): \delta 0.92(3 \mathrm{H}, \mathrm{t}, J=$ $7.3 \mathrm{~Hz}), 1.37(4 \mathrm{H}, \mathrm{m}), 1.70(2 \mathrm{H}, \mathrm{m}), 3.90\left(6 \mathrm{H}, \mathrm{s}, \mathrm{OCH}_{3}\right), 4.19(2 \mathrm{H}, \mathrm{t}, J=6.8 \mathrm{~Hz}), 6.30(1 \mathrm{H}, \mathrm{d}, J=16.0$ $\mathrm{Hz},-\mathrm{CO}-\mathrm{CH}=), 6.85(1 \mathrm{H}, \mathrm{d}, J=8.4 \mathrm{~Hz}, \mathrm{Ar}-\mathrm{H}), 7.05(1 \mathrm{H}, \mathrm{d}, J=1.6 \mathrm{~Hz}, \mathrm{Ar}-\mathrm{H}), 7.09(1 \mathrm{H}, \mathrm{dd}, J=8.4$, $1.6 \mathrm{~Hz}, \mathrm{Ar}-\mathrm{H}), 7.61(1 \mathrm{H}, \mathrm{d}, J=16.0 \mathrm{~Hz}, \mathrm{Ar}-\mathrm{CH}=\mathrm{C}) ;{ }^{13} \mathrm{C} \mathrm{NMR}\left(\mathrm{CDCl}_{3}, 125 \mathrm{MHz}\right): \delta 13.79\left(\mathrm{CH}_{3}\right)$, $22.28\left(\mathrm{CH}_{2}\right), 27.93\left(\mathrm{CH}_{2}\right), 28.35(\mathrm{CH} 2), 55.80\left(\mathrm{OCH}_{3}\right), 55.87\left(\mathrm{OCH}_{3}\right), 65.31\left(-\mathrm{OCH}_{2}-\right), 109.55$, 110.06, 115.78 (=C-CO-), 122.37, 127.29, 144.25 (Ar-C=), 149.12, 151.01, 167.24 (C = O). MS: m/z $279.1596(\mathrm{M}+1)$.

Hexyl (2E)-3-(3,4-dimethoxyphenyl)prop-2-enoate (8)

Yield 83\%; yellow pale oil; IR (KBr, cm $\left.{ }^{-1}\right): v \max 2957(\mathrm{C}-\mathrm{H}), 1704(\mathrm{C}=\mathrm{O}), 1600(\mathrm{C}=\mathrm{C}), 1512$ $\left(\mathrm{C}=\mathrm{C}_{\mathrm{Ar}}\right), 1270(\mathrm{C}-\mathrm{O}-\mathrm{C}), 1173((\mathrm{C}=\mathrm{O})-\mathrm{O}), 808\left(\mathrm{C}-\mathrm{H}_{\mathrm{Ar}}\right) ;{ }^{1} \mathrm{H} \mathrm{NMR}\left(\mathrm{CDCl}_{3}, 500 \mathrm{MHz}\right): \delta 0.91(3 \mathrm{H}, \mathrm{t}, J=$ $7.0 \mathrm{~Hz}), 1.33(4 \mathrm{H}, \mathrm{m}), 1.41(2 \mathrm{H}, \mathrm{m}), 1.70(2 \mathrm{H}, \mathrm{m}), 3.91\left(6 \mathrm{H}, \mathrm{s}, \mathrm{OCH}_{3}\right), 4.19(2 \mathrm{H}, \mathrm{t}, J=6.8 \mathrm{~Hz}), 6.31$ $(1 \mathrm{H}, \mathrm{d}, J=15.9 \mathrm{~Hz},-\mathrm{CO}-\mathrm{CH}=), 6.86(1 \mathrm{H}, \mathrm{d}, J=8.2 \mathrm{~Hz}, \mathrm{Ar}-\mathrm{H}), 7.06(1 \mathrm{H}, \mathrm{d}, J=1.8 \mathrm{~Hz}, \mathrm{Ar}-\mathrm{H}), 7.10$ $(1 \mathrm{H}, \mathrm{dd}, J=8.2,1.8 \mathrm{~Hz}, \mathrm{Ar}-\mathrm{H}), 7.62(1 \mathrm{H}, \mathrm{d}, \mathrm{J}=15.9 \mathrm{~Hz}, \mathrm{Ar}-\mathrm{CH}=\mathrm{C}) ;{ }^{13} \mathrm{C} \mathrm{NMR}\left(\mathrm{CDCl}_{3}, 125 \mathrm{MHz}\right): \delta$ $13.85\left(\mathrm{CH}_{3}\right), 22.43\left(\mathrm{CH}_{2}\right), 22.59\left(\mathrm{CH}_{2}\right), 28.74\left(\mathrm{CH}_{2}\right), 31.46\left(\mathrm{CH}_{2}\right), 55.84\left(\mathrm{OCH}_{3}\right), 55.91\left(\mathrm{OCH}_{3}\right), 64.76$ $\left(-\mathrm{OCH}_{2}-\right), 109.56,110.92,115.97$ (=C-CO-), 122.44, 127.33, 144.35 (Ar-C=), 149.07, 151.03, 167.07 $(\mathrm{C}=\mathrm{O}) . \mathrm{MS}: \mathrm{m} / \mathrm{z} 293.1753(\mathrm{M}+1)$.

Octyl (2E)-3-(3,4-dimethoxyphenyl)prop-2-enoate (9)

Yield 80\%; yellow pale oil; IR $\left(\mathrm{KBr}, \mathrm{cm}^{-1}\right): v_{\max } 2956(\mathrm{C}-\mathrm{H}), 1718(\mathrm{C}=\mathrm{O}), 1633(\mathrm{C}=\mathrm{C}), 1514$ $\left(\mathrm{C}=\mathrm{C}_{\mathrm{Ar}}\right), 1271(\mathrm{C}-\mathrm{O}-\mathrm{C}), 1176((\mathrm{C}=\mathrm{O})-\mathrm{O}), 802\left(\mathrm{C}-\mathrm{H}_{\mathrm{Ar}}\right) ;{ }^{1} \mathrm{H} \mathrm{NMR}\left(\mathrm{CDCl}_{3}, 500 \mathrm{MHz}\right): \delta 0.81(3 \mathrm{H}, \mathrm{t}, J=$ $6.6 \mathrm{~Hz}), 1.09-1.38(10 \mathrm{H}, \mathrm{m}), 1.61(2 \mathrm{H}, \mathrm{m}), 3.84\left(6 \mathrm{H}, \mathrm{s}, \mathrm{OCH}_{3}\right), 4.12(2 \mathrm{H}, \mathrm{t}, J=6.7 \mathrm{~Hz}), 6.24(1 \mathrm{H}, \mathrm{d}, J$ $=16.6 \mathrm{~Hz},-\mathrm{CO}-\mathrm{CH}=), 6.79(1 \mathrm{H}, \mathrm{d}, J=8.4 \mathrm{~Hz}, \mathrm{Ar}-\mathrm{H}), 6.99(1 \mathrm{H}, \mathrm{d}, J=1.3 \mathrm{~Hz}, \mathrm{Ar}-\mathrm{H}), 7.03(1 \mathrm{H}, \mathrm{dd}, J$ $=8.4,1.3 \mathrm{~Hz}, \mathrm{Ar}-\mathrm{H}), 7.55(1 \mathrm{H}, \mathrm{d}, J=16.6 \mathrm{~Hz}, \mathrm{Ar}-\mathrm{CH}=\mathrm{C}) ;{ }^{13} \mathrm{C} \mathrm{NMR}\left(\mathrm{CDCl}_{3}, 125 \mathrm{MHz}\right): \delta 14.52$ $\left(\mathrm{CH}_{3}\right), 23.09\left(\mathrm{CH}_{2}\right), 26.41\left(\mathrm{CH}_{2}\right), 29.15\left(\mathrm{CH}_{2}\right), 29.61\left(2 \mathrm{CH}_{2}\right), 32.20\left(\mathrm{CH}_{2}\right), 56.27\left(\mathrm{OCH}_{3}\right), 56.36$ $\left(\mathrm{OCH}_{3}\right), 65.21\left(-\mathrm{OCH}_{2}-\right), 109.88,111.39,112.69$ (3CH), 116.29 (=C-CO-), 123.01, 144.95 (Ar-C=), $167.68(\mathrm{C}=\mathrm{O})$. MS: $\mathrm{m} / \mathrm{z} 321.2023(\mathrm{M}+1)$.

Dodecyl (2E)-3-(3,4-dimethoxyphenyl)prop-2-enoate (10)

Yield 75\%; M.p. 55-58 ${ }^{\circ} \mathrm{C}$; IR (KBr, cm $\left.{ }^{-1}\right): v_{\max } 2939(\mathrm{C}-\mathrm{H}), 1697(\mathrm{C}=\mathrm{O}), 1625(\mathrm{C}=\mathrm{C}), 1512\left(\mathrm{C}=\mathrm{C}_{\mathrm{Ar}}\right)$, $1251(\mathrm{C}-\mathrm{O}-\mathrm{C}), 1161((\mathrm{C}=\mathrm{O})-\mathrm{O}), 813\left(\mathrm{C}-\mathrm{H}_{\mathrm{Ar}}\right) ;{ }^{1} \mathrm{H} \mathrm{NMR}(\mathrm{CDCl} 3,500 \mathrm{MHz}): \delta 0.85(3 \mathrm{H}, \mathrm{t}, \mathrm{J}=6.9 \mathrm{~Hz})$, 1.15-1.35 (18H, m), $1.53(2 \mathrm{H}, \mathrm{m}), 3.61(2 \mathrm{H}, \mathrm{t}, \mathrm{J}=6.67 \mathrm{~Hz}), 3.88\left(6 \mathrm{H}, \mathrm{s}, \mathrm{OCH}_{3}\right), 6.28(1 \mathrm{H}, \mathrm{d}, \mathrm{J}=16.4$ $\mathrm{Hz},-\mathrm{CO}-\mathrm{CH}=), 6.84(1 \mathrm{H}, \mathrm{d}, \mathrm{J}=8.3 \mathrm{~Hz}, \mathrm{Ar}-\mathrm{H}), 7.02(1 \mathrm{H}, \mathrm{d}, \mathrm{J}=1.1 \mathrm{~Hz}, \mathrm{Ar}-\mathrm{H}), 7.08(1 \mathrm{H}, \mathrm{dd}, \mathrm{J}=8.3$, $1.1 \mathrm{~Hz}, \mathrm{Ar}-\mathrm{H}), 7.61(1 \mathrm{H}, \mathrm{d}, \mathrm{J}=16.4 \mathrm{~Hz}, \mathrm{Ar}-\mathrm{CH}=\mathrm{C}) ;{ }^{13} \mathrm{C} \mathrm{NMR}\left(\mathrm{CDCl}_{3}, 125 \mathrm{MHz}\right): \delta 14.65\left(\mathrm{CH}_{3}\right)$, $23.06\left(\mathrm{CH}_{2}\right), 26.14\left(\mathrm{CH}_{2}\right), 29.75-32.02\left(6 \mathrm{CH}_{2}\right), 32.28\left(\mathrm{CH}_{2}\right), 33.27\left(\mathrm{CH}_{2}\right), 56.28\left(\mathrm{OCH}_{3}\right), 56.36$ $\left(\mathrm{OCH}_{3}\right), 63.45\left(-\mathrm{OCH}_{2}-\right), 109.86,111.39,113.34,115.85$ (=C-CO-), 122.88, 125.87, 137.42, 145.22 $(\mathrm{Ar}-\mathrm{C}=), 179.05(\mathrm{C}=\mathrm{O})$. MS: m/z $377.2632(\mathrm{M}+1)$.

Hexadecyl (2E)-3-(3,4-dimethoxyphenyl)prop-2-enoate (11)

Yield 87\%; M.p. 48-50 ${ }^{\circ} \mathrm{C}$; IR $\left(\mathrm{KBr}, \mathrm{cm}^{-1}\right): v_{\max } 2918(\mathrm{C}-\mathrm{H}), 1719(\mathrm{C}=\mathrm{O}), 1635(\mathrm{C}=\mathrm{C}), 1500\left(\mathrm{C}=\mathrm{C}_{\mathrm{Ar}}\right)$, $1271(\mathrm{C}-\mathrm{O}-\mathrm{C}), 1180((\mathrm{C}=\mathrm{O})-\mathrm{O}), 802\left(\mathrm{C}-\mathrm{H}_{\mathrm{Ar}}\right) ;{ }^{1} \mathrm{H} \mathrm{NMR}\left(\mathrm{CDCl}_{3}, 500 \mathrm{MHz}\right): \delta 0.89(3 \mathrm{H}, \mathrm{t}, J=7.1 \mathrm{~Hz})$, 1.15-1.47 (26H, m), $1.70(2 \mathrm{H}, \mathrm{m}), 3.91\left(6 \mathrm{H}, \mathrm{s}, \mathrm{OCH}_{3}\right), 4.20(2 \mathrm{H}, \mathrm{t}, \mathrm{J}=6.74 \mathrm{~Hz}), 6.31(1 \mathrm{H}, \mathrm{d}, \mathrm{J}=16.0$ $\mathrm{Hz},-\mathrm{CO}-\mathrm{CH}=), 6.87(1 \mathrm{H}, \mathrm{d}, J=8.3 \mathrm{~Hz}, \mathrm{Ar}-\mathrm{H}), 7.06(1 \mathrm{H}, \mathrm{d}, J=1.6 \mathrm{~Hz}, \mathrm{Ar}-\mathrm{H}), 7.11(1 \mathrm{H}, \mathrm{dd}, J=8.3$, $1.6 \mathrm{~Hz}, \mathrm{Ar}-\mathrm{H}), 7.63(1 \mathrm{H}, \mathrm{d}, J=16.0 \mathrm{~Hz}, \mathrm{Ar}-\mathrm{CH}=\mathrm{C}) ;{ }^{13} \mathrm{C} \mathrm{NMR}\left(\mathrm{CDCl}_{3}, 125 \mathrm{MHz}\right): \delta 14.08\left(\mathrm{CH}_{3}\right)$, $22.64\left(\mathrm{CH}_{2}\right), 25.70\left(\mathrm{CH}_{2}\right), 28.18-29.85\left(10 \mathrm{CH}_{2}\right), 31.91\left(\mathrm{CH}_{2}\right), 32.79\left(\mathrm{CH}_{2}\right), 55.84\left(\mathrm{OCH}_{3}\right), 55.93$ 
$\left(\mathrm{OCH}_{3}\right), 64.59\left(-\mathrm{OCH}_{2}-\right), 109.60,110.90,116.02$ (=C-CO-), 122.39, 127.45, 144.37 (Ar-C=), 150.95, 151.03, $167.14(\mathrm{C}=\mathrm{O})$. MS: $\mathrm{m} / \mathrm{z} 433.3318(\mathrm{M}+1)$.

Octadecyl (2E)-3-(3,4-dimethoxyphenyl)prop-2-enoate (12)

Yield 70\%; M.p. 154-158 ${ }^{\circ} \mathrm{C}$; IR $\left(\mathrm{KBr}, \mathrm{cm}^{-1}\right): v \max 2915$ (C-H), 1708 (C=O), 1620 (C=C), 1506 $\left(\mathrm{C}=\mathrm{C}_{\mathrm{Ar}}\right), 1274(\mathrm{C}-\mathrm{O}-\mathrm{C}), 1163((\mathrm{C}=\mathrm{O})-\mathrm{O}), 811\left(\mathrm{C}-\mathrm{H}_{\mathrm{Ar}}\right) ;{ }^{1} \mathrm{H} \mathrm{NMR}\left(\mathrm{CDCl}_{3}, 500 \mathrm{MHz}\right): \delta 0.87(3 \mathrm{H}, \mathrm{t}, J=$ $7.1 \mathrm{~Hz}), 1.18-1.39(30 \mathrm{H}, \mathrm{m}), 1.55(2 \mathrm{H}, \mathrm{m}), 3.62(2 \mathrm{H}, \mathrm{t}, J=6.6 \mathrm{~Hz}), 3.92\left(6 \mathrm{H}, \mathrm{s}, \mathrm{OCH}_{3}\right), 6.38(1 \mathrm{H}, \mathrm{d}, J$ $=16.0 \mathrm{~Hz},-\mathrm{CO}-\mathrm{CH}=), 6.88(1 \mathrm{H}, \mathrm{d}, J=8.3 \mathrm{~Hz}, \mathrm{Ar}-\mathrm{H}), 7.07(1 \mathrm{H}, \mathrm{d}, J=1.8 \mathrm{~Hz}, \mathrm{Ar}-\mathrm{H}), 7.15(1 \mathrm{H}, \mathrm{dd}, J$ $=8.3,1.8 \mathrm{~Hz}, \mathrm{Ar}-\mathrm{H}), 7.78(1 \mathrm{H}, \mathrm{d}, J=16.0 \mathrm{~Hz}, \mathrm{Ar}-\mathrm{CH}=\mathrm{C}) ;{ }^{13} \mathrm{C} \mathrm{NMR}\left(\mathrm{CDCl}_{3}, 125 \mathrm{MHz}\right): \delta 14.06$ $\left(\mathrm{CH}_{3}\right), 22.60\left(\mathrm{CH}_{2}\right), 25.70\left(\mathrm{CH}_{2}\right), 29.30\left(\mathrm{CH}_{2}\right), 29.40-29.80\left(11 \mathrm{CH}_{2}\right), 31.89\left(\mathrm{CH}_{2}\right), 32.77\left(\mathrm{CH}_{2}\right), 55.90$ $\left(\mathrm{OCH}_{3}\right), 55.98\left(\mathrm{OCH}_{3}\right), 63.03\left(-\mathrm{OCH}_{2}\right), 109.96,111.08,114.35$ (=C-CO-), 123.55, 126.74, 148.47 (Ar-C=), 149.33, 151.95, $162.84(\mathrm{C}=\mathrm{O}) . \mathrm{MS}: \mathrm{m} / \mathrm{z} 461.3631(\mathrm{M}+1)$.

Hexadecyl (2E)-3-(2,3-dimethoxyphenyl)prop-2-enoate (13)

Yield 60\%; M.p. $41-43{ }^{\circ} \mathrm{C}$; IR (KBr, cm $\left.{ }^{-1}\right): v_{\max } 2920(\mathrm{C}-\mathrm{H}), 1719(\mathrm{C}=\mathrm{O}), 1635(\mathrm{C}=\mathrm{C}), 1515\left(\mathrm{C}=\mathrm{C}_{\mathrm{Ar}}\right)$, $1270(\mathrm{C}-\mathrm{O}-\mathrm{C}), 1180((\mathrm{C}=\mathrm{O})-\mathrm{O}), 802\left(\mathrm{C}-\mathrm{H}_{\mathrm{Ar}}\right) ;{ }^{1} \mathrm{H} \mathrm{NMR}\left(\mathrm{CDCl}_{3}, 500 \mathrm{MHz}\right): \delta 0.89(3 \mathrm{H}, \mathrm{t}, J=7.1 \mathrm{~Hz})$, 1.13-1.50 (26H, m), $1.72(2 \mathrm{H}, \mathrm{m}), 3.87\left(3 \mathrm{H}, \mathrm{s}, \mathrm{OCH}_{3}\right), 3.89\left(3 \mathrm{H}, \mathrm{s}, \mathrm{OCH}_{3}\right), 4.21(2 \mathrm{H}, \mathrm{t}, J=6.7 \mathrm{~Hz})$, $6.49(1 \mathrm{H}, \mathrm{d}, J=16.2 \mathrm{~Hz},-\mathrm{CO}-\mathrm{CH}=), 6.94(1 \mathrm{H}, \mathrm{d}, J=8.0 \mathrm{~Hz}, \mathrm{Ar}-\mathrm{H}), 7.06(1 \mathrm{H}, \mathrm{t}, J=8.0 \mathrm{~Hz}, \mathrm{Ar}-\mathrm{H})$, $7.16(1 \mathrm{H}, \mathrm{d}, J=8.0 \mathrm{~Hz}, \mathrm{Ar}-\mathrm{H}), 8.00(1 \mathrm{H}, \mathrm{d}, J=16.2 \mathrm{~Hz}, \mathrm{Ar}-\mathrm{CH}=\mathrm{C}) ;{ }^{13} \mathrm{C} \mathrm{NMR}\left(\mathrm{CDCl}_{3}, 125 \mathrm{MHz}\right): \delta$ $14.04\left(\mathrm{CH}_{3}\right), 22.68\left(\mathrm{CH}_{2}\right), 25.95\left(\mathrm{CH}_{2}\right), 28.74\left(\mathrm{CH}_{2}\right), 29.04-29.90\left(10 \mathrm{CH}_{2}\right), 31.86\left(\mathrm{CH}_{2}\right), 55.88$ $\left(\mathrm{OCH}_{3}\right), 61.26\left(\mathrm{OCH}_{3}\right), 64.66\left(-\mathrm{OCH}_{2}-\right), 113.85$ (=C-CO-), 119.21, 119.65, 124.11, 128.69, 139.29 $(\mathrm{Ar}-\mathrm{C}=), 148.50,153.15,167.19(\mathrm{C}=\mathrm{O}) . \mathrm{MS}: \mathrm{m} / \mathrm{z} 433.3318(\mathrm{M}+1)$.

\section{Hexadecyl (2E)-3-(2,5-dimethoxyphenyl)prop-2-enoate (14)}

Yield 85\%; M.p. 63-65 ${ }^{\circ} \mathrm{C}$; IR (KBr, cm $\left.{ }^{-1}\right): v_{\max } 2918(\mathrm{C}-\mathrm{H}), 1710(\mathrm{C}=\mathrm{O}), 1630(\mathrm{C}=\mathrm{C}), 1495\left(\mathrm{C}=\mathrm{C}_{\mathrm{Ar}}\right)$, $1219(\mathrm{C}-\mathrm{O}-\mathrm{C}), 1177((\mathrm{C}=\mathrm{O})-\mathrm{O}), 802\left(\mathrm{C}-\mathrm{H}_{\mathrm{Ar}}\right) ;{ }^{1} \mathrm{H} \mathrm{NMR}\left(\mathrm{CDCl}_{3}, 500 \mathrm{MHz}\right): \delta 0.89(3 \mathrm{H}, \mathrm{t}, J=7.0 \mathrm{~Hz})$, 1.22-1.44 (26H, m), $1.72(2 \mathrm{H}, \mathrm{m}), 3.80\left(3 \mathrm{H}, \mathrm{s}, \mathrm{OCH}_{3}\right), 3.85\left(3 \mathrm{H}, \mathrm{s}, \mathrm{OCH}_{3}\right), 4.20(2 \mathrm{H}, \mathrm{t}, J=6.81 \mathrm{~Hz})$, $6.50(1 \mathrm{H}, \mathrm{d}, J=16.2 \mathrm{~Hz},-\mathrm{CO}-\mathrm{CH}=), 6.85(1 \mathrm{H}, \mathrm{d}, J=9.0 \mathrm{~Hz}, \mathrm{Ar}-\mathrm{H}), 6.92(1 \mathrm{H}, \mathrm{dd}, J=9.0,3.0 \mathrm{~Hz})$, $7.06(1 \mathrm{H}, \mathrm{d}, J=3.0 \mathrm{~Hz}, \mathrm{Ar}-\mathrm{H}), 7.97(1 \mathrm{H}, \mathrm{d}, J=16.2 \mathrm{~Hz}, \mathrm{Ar}-\mathrm{CH}=\mathrm{C}) ;{ }^{13} \mathrm{C} \mathrm{NMR}\left(\mathrm{CDCl}_{3}, 125 \mathrm{MHz}\right): \delta$ $14.08\left(\mathrm{CH}_{3}\right), 22.68\left(\mathrm{CH}_{2}\right), 25.96\left(\mathrm{CH}_{2}\right), 28.77\left(\mathrm{CH}_{2}\right), 29.17-29.97\left(10 \mathrm{CH}_{2}\right), 31.90\left(\mathrm{CH}_{2}\right), 55.09$ $\left(\mathrm{OCH}_{3}\right), 55.77\left(\mathrm{OCH}_{3}\right), 64.56\left(-\mathrm{OCH}_{2}\right), 112.46,113.24,116.99,119.04,124.07$ (=C-CO-), 139.67 $(\mathrm{Ar}-\mathrm{C}=), 152.77,153.56,167.37(\mathrm{C}=\mathrm{O}) . \mathrm{MS}: \mathrm{m} / \mathrm{z} 433.3318(\mathrm{M}+1)$.

Hexadecyl (2E)-3-(4-methoxyphenyl)prop-2-enoate (15)

Yield 78\%; M.p. 50-55 ${ }^{\circ} \mathrm{C}$; IR $\left(\mathrm{KBr}, \mathrm{cm}^{-1}\right): v_{\max } 2915(\mathrm{C}-\mathrm{H}), 1707(\mathrm{C}=\mathrm{O}), 1638(\mathrm{C}=\mathrm{C}), 1517\left(\mathrm{C}=\mathrm{C}_{\mathrm{Ar}}\right)$, $1268(\mathrm{C}-\mathrm{O}-\mathrm{C}), 1182((\mathrm{C}=\mathrm{O})-\mathrm{O}), 826\left(\mathrm{C}-\mathrm{H}_{\mathrm{Ar}}\right) ;{ }^{1} \mathrm{H} \mathrm{NMR}\left(\mathrm{CDCl}_{3}, 500 \mathrm{MHz}\right): \delta 0.89(3 \mathrm{H}, \mathrm{t}, J=7.1 \mathrm{~Hz})$, 1.19-1.46 (26H, m), $1.70(2 \mathrm{H}, \mathrm{m}), 3.84\left(3 \mathrm{H}, \mathrm{s}, \mathrm{OCH}_{3}\right), 4.20(2 \mathrm{H}, \mathrm{t}, J=6.9 \mathrm{~Hz}), 6.32(1 \mathrm{H}, \mathrm{d}, J=16.0$ $\mathrm{Hz},-\mathrm{CO}-\mathrm{CH}=), 6.92(2 \mathrm{H}, \mathrm{d}, J=8.7 \mathrm{~Hz}, \mathrm{Ar}-\mathrm{H}), 7.48(2 \mathrm{H}, \mathrm{d}, J=8.7 \mathrm{~Hz}, \mathrm{Ar}-\mathrm{H}), 7.64(1 \mathrm{H}, \mathrm{d}, J=16.0$ $\mathrm{Hz}, \mathrm{Ar}-\mathrm{CH}=\mathrm{C}) ;{ }^{13} \mathrm{C} \mathrm{NMR}\left(\mathrm{CDCl}_{3}, 125 \mathrm{MHz}\right): \delta 14.06\left(\mathrm{CH}_{3}\right), 22.64\left(\mathrm{CH}_{2}\right), 25.97\left(\mathrm{CH}_{2}\right), 28.75\left(\mathrm{CH}_{2}\right)$, 29.20-29.82 $\left(10 \mathrm{CH}_{2}\right), 31.89\left(\mathrm{CH}_{2}\right), 55.32\left(\mathrm{OCH}_{3}\right), 64.58\left(-\mathrm{OCH}_{2}-\right), 114.24,115.79$ (=C-CO-), 127.24, 129.59, 144.11 (Ar-C=), 161.31, 167.33 (C=O). MS: m/z 403.3212 (M+1).

Hexadecyl 3-(4-methoxyphenyl)propanoate (16)

Yield 70\%; yellow pale oil; IR (KBr, $\left.\mathrm{cm}^{-1}\right): v_{\max } 2929(\mathrm{C}-\mathrm{H}), 1735(\mathrm{C}=\mathrm{O}), 1515\left(\mathrm{C}=\mathrm{C}_{\mathrm{Ar}}\right), 1247(\mathrm{C}-\mathrm{O}-$ C), $1175((\mathrm{C}=\mathrm{O})-\mathrm{O}), 835\left(\mathrm{C}-\mathrm{H}_{\mathrm{Ar}}\right) ;{ }^{1} \mathrm{H} \mathrm{NMR}\left(\mathrm{CDCl}_{3}, 500 \mathrm{MHz}\right): \delta 0.90(3 \mathrm{H}, \mathrm{t}, J=7.1 \mathrm{~Hz}), 1.23-1.35$ $(26 \mathrm{H}, \mathrm{m}), 1.60(2 \mathrm{H}, \mathrm{m}), 2.60(2 \mathrm{H}, \mathrm{t}, J=7.8), 2.90,(2 \mathrm{H}, \mathrm{t}, J=7.8), 3.79\left(3 \mathrm{H}, \mathrm{s}, \mathrm{OCH}_{3}\right), 4.07(2 \mathrm{H}, \mathrm{t}, J=$ $7.1 \mathrm{~Hz}), 6.83(2 \mathrm{H}, \mathrm{d}, J=8.3 \mathrm{~Hz}, \mathrm{Ar}-\mathrm{H}), 7.13(2 \mathrm{H}, \mathrm{d}, J=8.3 \mathrm{~Hz}, \mathrm{Ar}-\mathrm{H}) .{ }^{13} \mathrm{C} \mathrm{NMR}\left(\mathrm{CDCl}_{3}, 125 \mathrm{MHz}\right)$ : 
$\delta 14.10\left(\mathrm{CH}_{3}\right), 22.60\left(\mathrm{CH}_{2}\right), 25.87\left(\mathrm{CH}_{2}\right), 28.60\left(\mathrm{CH}_{2}\right), 29.01-30.45\left(11 \mathrm{CH}_{2}\right), 31.94\left(\mathrm{CH}_{2}\right), 36.16$ $\left(\mathrm{CH}_{2}\right), 55.14\left(\mathrm{OCH}_{3}\right), 64.54\left(-\mathrm{OCH}_{2}\right), 113.83, \quad 129.18,132.32,158.04,172.97(\mathrm{C}=\mathrm{O}) . \mathrm{MS}: \mathrm{m} / \mathrm{z}$ $405.3369(\mathrm{M}+1)$.

Hexadecyl (2E)-3-phenylprop-2-enoate (17)

Yield 94\%; M.p. 35-36 ${ }^{\circ} \mathrm{C}$; IR (KBr, cm $\left.{ }^{-1}\right): v_{\max } 2952(\mathrm{C}-\mathrm{H}), 1714(\mathrm{C}=\mathrm{O}), 1640(\mathrm{C}=\mathrm{C}), 1475\left(\mathrm{C}=\mathrm{C}_{\mathrm{Ar}}\right)$, $1177((\mathrm{C}=\mathrm{O})-\mathrm{O}), 801\left(\mathrm{C}-\mathrm{H}_{\mathrm{Ar}}\right) ;{ }^{1} \mathrm{H} \mathrm{NMR}\left(\mathrm{CDCl}_{3}, 500 \mathrm{MHz}\right): \delta 0.89(3 \mathrm{H}, \mathrm{t}, J=7.0 \mathrm{~Hz}), 1.19-1.48(26 \mathrm{H}$, $\mathrm{m}), 1.72(2 \mathrm{H}, \mathrm{m}), 4.21(2 \mathrm{H}, \mathrm{t}, J=6.8 \mathrm{~Hz}), 6.35(1 \mathrm{H}, \mathrm{d}, J=16.0 \mathrm{~Hz},-\mathrm{CO}-\mathrm{CH}=), 7.39(3 \mathrm{H}, \mathrm{m}, \mathrm{Ar}-\mathrm{H})$, $7.53(2 \mathrm{H}, \mathrm{dd}, J=7.0,2.2 \mathrm{~Hz}, \mathrm{Ar}-\mathrm{H}), 7.69(1 \mathrm{H}, \mathrm{d}, J=16.0 \mathrm{~Hz}, \mathrm{Ar}-\mathrm{CH}=\mathrm{C}) ;{ }^{13} \mathrm{C} \mathrm{NMR}\left(\mathrm{CDCl}_{3}, 125\right.$ $\mathrm{MHz}): \delta 14.06\left(\mathrm{CH}_{3}\right), 22.66\left(\mathrm{CH}_{2}\right), 26.01\left(\mathrm{CH}_{2}\right), 28.72\left(\mathrm{CH}_{2}\right), 29.22-29.91\left(10 \mathrm{CH}_{2}\right), 31.89\left(\mathrm{CH}_{2}\right)$, $64.66\left(-\mathrm{OCH}_{2}-\right), 118.32$ (=C-CO-), 127.97, 128.82, 130.10, 134.45, 144.47 (Ar-C=), 167 (C = O). MS: $\mathrm{m} / \mathrm{z} 373.3107(\mathrm{M}+1)$.

\section{Octyl 3-(3,4-dihydroxyphenyl)prop-2-enoate (18)}

Yield 72\%; M.p. 101-104 ${ }^{\circ} \mathrm{C}$; IR (KBr, cm $\left.{ }^{-1}\right): v_{\max } 3318(\mathrm{OH}), 2920(\mathrm{C}-\mathrm{H}), 1683(\mathrm{C}=\mathrm{O}), 1604(\mathrm{C}=\mathrm{C})$, $1442\left(\mathrm{C}=\mathrm{C}_{\mathrm{Ar}}\right), 1282(\mathrm{C}-\mathrm{O}-\mathrm{C}), 1178((\mathrm{C}=\mathrm{O})-\mathrm{O}), 815\left(\mathrm{C}-\mathrm{H}_{\mathrm{Ar}}\right) ;{ }^{1} \mathrm{H}$ NMR $\left(\mathrm{DMSO}_{6}, 500 \mathrm{MHz}\right): \delta 0.79$ $(3 \mathrm{H}, \mathrm{t}, J=6.5 \mathrm{~Hz}), 1.03-1.31(10 \mathrm{H}, \mathrm{m}), 1.53(2 \mathrm{H}, \mathrm{m}), 4.01(2 \mathrm{H}, \mathrm{t}, J=6.0 \mathrm{~Hz}), 4.43(2 \mathrm{H}, \mathrm{m}, \mathrm{OH}), 6.17$ $(1 \mathrm{H}, \mathrm{d}, J=15.9 \mathrm{~Hz},-\mathrm{CO}-\mathrm{CH}=), 6.75(1 \mathrm{H}, \mathrm{d}, J=8.2 \mathrm{~Hz}, \mathrm{Ar}-\mathrm{H}), 6.83(1 \mathrm{H}, \mathrm{d}, J=8.2 \mathrm{~Hz}, \mathrm{Ar}-\mathrm{H}), 7.02$ $(1 \mathrm{H}, \mathrm{s}, \mathrm{Ar}-\mathrm{H}), 7.42(1 \mathrm{H}, \mathrm{d}, J=15.9 \mathrm{~Hz}, \mathrm{Ar}-\mathrm{CH}=\mathrm{C}) ;{ }^{13} \mathrm{C}$ NMR $\left(\mathrm{DMSO}-\mathrm{D}_{6}, 125 \mathrm{MHz}\right): \delta 13.65\left(\mathrm{CH}_{3}\right)$, $22.35\left(\mathrm{CH}_{2}\right), 25.73\left(\mathrm{CH}_{2}\right), 28.50\left(\mathrm{CH}_{2}\right), 28.98\left(\mathrm{CH}_{2}\right), 31.41\left(\mathrm{CH}_{2}\right), 45.96\left(\mathrm{CH}_{2}\right), 63.98\left(-\mathrm{OCH}_{2^{-}}\right)$, 111.65, 121.61 (=C-CO-), 125.93 (2C), 145.21 (2C), 148.20 (Ar-C=C), 167.68, 167.01 (C = O). MS: $\mathrm{m} / \mathrm{z} 293.1732(\mathrm{M}+1)$.

\section{Biological activity assays}

The compounds were subjected to in vitro leishmanicidal activity on amastigotes of L. panamensis and cytotoxic activity on mammalian cells.

In vitro cytotoxic activity in mammalian cells

The cytotoxic activity of the compounds was assessed based on the viability of the human promonocytic cell line U937 (ATCC CRL-1593.2 ${ }^{\mathrm{TM}}$ ) evaluated by the MTT (3-(4,5-dimethylthiazol-2yl)-2,5-diphenyltetrazolium bromide) method as described by Robledo et al. (2005). In brief, cells were grown in 96-well cell-culture dishes at a concentration of 100,000 cells/ml in RPMI-1640 supplemented with $10 \%$ FBS and the corresponding concentrations of the compounds, starting at 200 $\mathrm{lg} / \mathrm{ml}$ in duplicate. The cells were incubated at $37^{\circ} \mathrm{C}$ with $5 \% \mathrm{CO}_{2}$ for $72 \mathrm{~h}$ in the presence of the compounds, and then the effect was determined using MTT assay, incubating at $37^{\circ} \mathrm{C}$ for $3 \mathrm{~h}$. The effect of the compounds was determined by measuring the activity of the mitochondrial dehydrogenase by adding $10 \mathrm{ll} /$ well of MTT solution $(0.5 \mathrm{mg} / \mathrm{ml})$ and incubating at $37^{\circ} \mathrm{C}$ for $3 \mathrm{~h}$. The reaction was stopped by adding a $50 \%$ isopropanol solution with $10 \%$ sodium dodecyl sulfate for 30 min. Cell viability was determined based on the quantity of formazan produced, which was measured at $570 \mathrm{~nm}$ in a Bio-Rad ELISA. Cultured cells in the absence of extracts were used as viability controls; Amphotericin B was used as cytotoxicity control. The results are expressed as the Lethal Concentration $50\left(\mathrm{LC}_{50}\right)$ calculated by the Probit method (Finney, 1971).

In vitro leishmanicidal activity on axenic and intracellular amastigotes 
Axenic and intracellular amastigotes of GFP-transfected L. (V.) panamensis strain (MHOM/CO/87/UA140epirGFP) were used for the in vitro testing of leishmanicidal activity of the cinnamic acid esters derivatives.

Activity against axenic amastigotes

The respective ability of the cinnamic acid esters to kill axenic amastigotes of L. (V.) panamensis was determined based on the viability of the parasites evaluated by the MTT (3-(4,5-dimethylthiazol-2-yl)2,5-diphenyltetrazolium bromide) method as described previously (Taylor et al., 2010). In short, parasites were cultivated in Schneider's medium pH 5.4 supplemented with $20 \%$ heat inactivated FBS for 3 days at $32^{\circ} \mathrm{C}$. Afterward, they were harvested, washed, and resuspended at $2 \times 10^{6}$ axenic amastigotes $/ \mathrm{ml}$ in fresh medium. Each well of a 96-well plate was seeded with $100 \mu \mathrm{l}$ of each parasite suspension (in duplicate), and $100 \mu \mathrm{l}$ of each concentration of the test compound was added, starting at $100 \mu \mathrm{g} / \mathrm{ml}$. Plates were incubated at $32^{\circ} \mathrm{C}$. After $72 \mathrm{~h}$ of incubation, the effect of drugs was determined by adding $10 \mu \mathrm{l} /$ well of MTT and incubating at $32^{\circ} \mathrm{C}$ for $3 \mathrm{~h}$. The reaction was stopped, and the quantity of formazan produced was measured with a Bio-Rad ELISA reader set at $570 \mathrm{~nm}$. Parasites cultivated in the absence of the compound but maintained under the same conditions were used as controls for growth and viability. Parasites cultivated in the presence of Amphotericin B were used as controls for leishmanicidal activity.

Activity against intracellular amastigotes

The effects of cinnamic acid esters against intracellular amastigotes of L. (V.) panamensis were evaluated by flow cytometry using the methodology described by Varela et al. (2009). In brief, U937 cells were dispensed in 24-well plates at a concentration of 300,000 cells/well, which were treated with $1 \mu \mathrm{M}$ of Phorbol Myristate Acetate (PMA) for $48 \mathrm{~h}$ at $37^{\circ} \mathrm{C}$, after which they were infected with promastigotes of L. (V.) panamensis in stationary growth phase (day 5) in modified NNN medium, a proportion of $1: 25$ cell/parasite, after $3 \mathrm{~h}$ of incubation at $34^{\circ} \mathrm{C}$ in $5 \% \mathrm{CO}_{2}$ non-internalized parasites were washed, and incubated again at $34^{\circ} \mathrm{C}$ and $5 \% \mathrm{CO}_{2}$ to allow differentiation to amastigotes form. After $24 \mathrm{~h}$ of incubation, the compounds with the appropriate dilution, not exceeding the $\mathrm{LC}_{50}$, were added. Infected and treated cells were maintained at $34^{\circ} \mathrm{C}$ and $5 \% \mathrm{CO}_{2}$ for $72 \mathrm{~h}$. The leishmanicidal effect was measured in a flow cytometer at $488 \mathrm{~nm}$ of excitation and $525 \mathrm{~nm}$ of emission, and determined as described by Varela et al. (2009). The results are expressed as the Effective Concentration 50 (EC50) calculated by the Probit statistical method. The data are the averages of three independent experiments conducted in duplicate. Infected but untreated cells were used as control of viability. In addition, infected cells exposed to Amphotericin B were used as leishmanicidal activity controls. The Selectivity Index (SI) was calculated by dividing the cytotoxic activity between the leishmanicidal activity $\left(\mathrm{SI}=\mathrm{LC}_{50} / \mathrm{EC}_{50}\right)$.

Acknowledgment The authors thank Dr. Javier Garcés for his help during the performance of this work. We acknowledge support by the Universidad de Antioquia (Estrategia de Sostenibilidad 20132014) 


\section{References}

Almajano M.P, Carbó R, Delgado M.E, Gordon M.H (2007) Effect of pH on the Antimicrobial Activity and Oxidative Stability of Oil-in-Water Emulsions containing Caffeic Acid. J Food Sci 72:C258-263

Barrett M, Gilbert I (2002) Perspectives for new drugs against trypanosomiasis and leishmaniasis. Curr Top Med Chem 2:471-482

Bowles BL, Miller A.J (1994) Caffeic Acid Activity against Clostridium botulinum Spores. J Food Sci 59:905-908

Buck S, Hardouin C, Ichikawa S, Soenen D, Gauss C, Hwang I, Swingle M, Bonness K, Honkanen R, Boger D (2003) Fundamental Role of the Fostriecin Unsaturated Lactone and Implications for Selective Protein Phosphatase Inhibition. J Am Chem Soc 125:15694-15695

Cabanillas B, Le Lamer A.C, Castillo D, Arevalo J, Rojas R, Odonne G, Bourdy G, Moukarzel B, Sauvain M, Fabre N (2010) Caffeic Acid Esters and Lignans from Piper sanguineispicum. J Nat Prod 73:1884-1890

Cardona W, Quiñones W, Robledo S, Vélez I, Murga J, et. al., (2006) Antiparasite and antimycobacterial activity of passifloricin analogues. Tetrahedron 62:4086-4092

Croft S, Coombs G (2003) Leishmaniasis-current chemotherapy and recent advances in the search for novel drugs. Trends Parasitol 19:502-508

Da Cunha FM, Duma D, Assreuy J, Buzzi FC, Niero R, Campos MM, Calixto JB (2004) Caffeic acid derivatives: in vitro and in vivo anti-inflammatory properties. Free Radic Res 38:1241-1253

De Campos F, Franzoi C, Antonini G, Fracasso M, Cechinel V, Yunes R, Niero R (2009) Antinociceptive properties of caffeic acid derivatives in mice. Eur J Med Chem 44:4596-4602

De Fatima A, Kohn L, Antonio M, De Carvalho J, Pilli R (2006) Cytotoxic activity of (S)goniothalamin and analogues against human cancer cells. Bioorg Med Chem 14:622-631

De P, Baltas M, Bedos-Belval F (2011) Cinnamic acid derivatives as anticancer agents-a review. Curr Med Chem 18:1672-1703

Desjeux P (2004) Leishmaniasis: current situation and new perspectives. Comp Immunol Microbiol Infect Dis 27:305-318

Faraut-Gambarelli F, Piarroux R, Deniau M, Giusiano B, Marty P, Michel G, Faugere B, Dumon H (1997) In vitro and in vivo Resistance of Leishmania infantum to Meglumine Antimoniate: Study of 37 Strains Collected from Patients with Visceral Leishmaniasis. Antimicrob Agents Chemother 41:827-830

Finney JD (1971) Probit Analysis (3rd edition) Cambridge University Press, Cambridge, UK Gupta M, Purnima B (2007) Tetrabutylammoniumbromide mediated Knoevenagel condensation in water: synthesis of cinnamic acids. ARKIVOC 1:94-98

Handman E (1999) Cell biology of Leishmania. Adv Parasitol 44:1-39

Hung C, Tsai W, Kuo L.Y, Kuo Y (2005) Evaluation of caffeic acid amide analogues as anti-platelet aggregation and anti-oxidative agents. Bioorg Med Chem 13:1791-1797

Jayaprakasam B, Vanisree M, Zhang Y, Dewitt D, Nair M (2006) Impact of Alkyl Esters of Caffeic and Ferulic Acids on Tumor Cell Proliferation, Cyclooxygenase Enzyme, and Lipid Peroxidation. J Agric Food Chem 54:5375-5381

Kikuzaki H, Hisamoto M, Hirose K, Akiyama K, Taniguchi H (2002) Antioxidant properties of ferulic acid and its related compounds. J Agric Food Chem 50:2161-2168

King P.J, Ma G, Miao W, Jia Q, McDougall B.R, Reinecke M.G, Cornell C, Kuan J, Kim T.R, Robinson W.E (1999) Structure-activity relationships: analogues of the dicaffeoylquinic and dicaffeoyltartaric acids as potent inhibitors of human immunodeficiency virus type 1 integrase and replication. J Med Chem 42:497-509

Murray HW, Berman JD, Davies CR, Saravia NG (2005) Advances in leishmaniasis. 


\section{Lancet 366:1561-1577}

Narasimhan B, Belsare D, Pharande D, Mourya V, Dhake A (2004) Esters, amides and substituted derivatives of cinnamic acid: synthesis, antimicrobial activity and QSAR investigations. Eur $\mathbf{J}$ Med Chem 39:827-834

Noriaki K, Yukari K, Kazuya I, Kyo M, Tokio F (2005) In Vitro Antibacterial, antimutagenic and antiinfluenza virus activity of caffeic acid phenethyl ester. Biocontrol Sci 10:155-161

Olliaro P, Bryceson A (1993) Practical progress and new drugs for changing patterns of Leishmaniasis. Parasitol Today 9:323-328

Ouellette M, Drummelsmith J, Papadopoulou B (2004) Leishmaniasis: drugs in the clinic, resistance and new developments. Drug Resist Updat 7:257-266

Radtke O.A, Foo L.Y, Lu Y, Kiderlen A, Kolodziej H (2003) Evaluation of Sage Phenolics for Their Antileishmanial Activity and Modulatory Effects on Interleukin-6, Interferon and Tumour Necrosis Factor- $\alpha$-Release in RAW 264.7 Cells. Z Naturforsch C 58(5-6):395-400

Rajan P, Vedernikova I, Cos P, Berghe D.V, Augustynsa K, Haemersa A (2001) Synthesis and evaluation of caffeic acid amides as antioxidants. Bioorg Med Chem Lett 11:215-217

Robledo S, Osorio E, Jaramillo L (2005) In vitro and in vivo cytotoxicities and antileishmanial activities of thymol and hemisynthetic derivatives. Antimic Agents Chemoth 49:1652-1655

Robledo S.M, Valencia A.Z, Saravia N.G (1999) Sensitivity to Glucantime of Leishmania Viannia isolated from patiens prior to treatment. J Parasitol 85:360-366

Son S, Lewis B.A (2002) Free radical scavenging and antioxidative activity of caffeic acid amide and ester analogues: structure-activity relationship. J Agric Food Chem 50:468-472

Valenta C, Schwarz E, Bernkop-Schnurch A (1998) Lysozyme-caffeic acid conjugates: possible novel preservatives for dermal formulations. Int J Pharm 174:125-132

Varela MRE, Muñoz DL, Robledo SM, Kolli BK, Dutta S, Chang KP, Muskus C (2009) Leishmania (Viannia) panamensis: an in vitro assay using the expression of GFP for screening of antileishmanial drug. Exp Parasitol 122:134-9

Weninger B, Robledo S, Arango G, Deharo E, Aragón R, Muñoz V, Callapa J, Lobstein A, Anton R, (2001) Antiprotozoal activities of Colombian plants. J Ethnopharmacol 78:193-200

Wolday D, Berhe N, Akuffo H, Britton S (1999) Leishmania-HIV interation: immunopathogenic mechanism. Parasitol Today 15:182-187 